\title{
Review
}

\section{Diastolic Dysfunction in Hypertensive Hearts: Roles of Perivascular Inflammation and Reactive Myocardial Fibrosis}

\author{
Hisashi KAI, Fumitaka KUWAHARA, Keisuke TOKUDA, and Tsutomu IMAIZUMI
}

\begin{abstract}
There is increasing evidence that myocardial fibrosis plays a role in the pathogenesis of diastolic dysfunction in hypertensive heart disease. However, it has been difficult to explore the mechanisms of isolated diastolic dysfunction in hypertensive hearts because of the lack of adequate animal models. Recently, we demonstrated that Wistar rats with a suprarenal aortic constriction (AC) can be used as a model of cardiac hypertrophy associated with preserved systolic, but impaired diastolic function without overt congestive heart failure. In this model, acute pressure elevation induces reactive myocardial fibrosis (perivascular fibrosis followed by intermuscular interstitial fibrosis) and myocyte/left ventricular (LV) hypertrophy. Perivascular macrophage infiltration, which is mediated by monocyte chemoattractant protein-1 (MCP-1) and intercellular adhesion molecule-1, exerts a key role in myocardial fibrosis, but not in myocyte/LV hypertrophy. Transforming growth factor (TGF)- $\beta$ is crucial for reactive fibrosis in AC rats. MCP-1 function blocking not only inhibits macrophage infiltration and TGF- $\beta$ induction but also prevents reactive fibrosis and diastolic dysfunction, without affecting blood pressure, myocyte/LV hypertrophy, or systolic function. Accordingly, a substantial role of inflammation is indicated in myocardial fibrosis and diastolic dysfunction in hypertensive hearts. Currently, the precise mechanisms whereby acute pressure elevation triggers inflammation remain unknown, but it is likely that activation of the tissue angiotensin system is involved in the induction of the inflammatory process. (Hypertens Res 2005; 28: 483-490)
\end{abstract}

Key Words: hypertension, myocardial fibrosis, inflammation, macrophages, angiotensin II

\section{Introduction}

In one-third to one-half of patients who are diagnosed as having congestive heart failure (CHF), systolic function of the left ventricle (LV) is preserved, indicating that diastolic dysfunction is a major pathophysiological abnormality in these patients (1-3). Diastolic dysfunction is highly prevalent, particularly in hypertensive elderly patients $(3,4)$. Patients with diastolic dysfunction are susceptive to pulmonary congestion, because the LV end-diastolic pressure (LVEDP) increases upon mild effort or mild volume overload (5). This is the major cause of impaired quality of life in patients with diastolic dysfunction. In some patients with diastolic dysfunction, impairment of systolic function also progresses and eventually leads to death. Furthermore, recent epidemiological studies showed that the prognosis of CHF patients with normal systolic function is not always better than that in $\mathrm{CHF}$ patients

From the Third Department of Internal Medicine and Cardiovascular Research Institute, Kurume University School of Medicine, Kurume, Japan.

This study was supported in part by a grant for the Academic Frontier Project and Grants-in-Aid for Scientific Research (to F.K., H.K.) from the Ministry of Education, Science, Sports, Culture, and Technology, Japan; and by research grants from the Sharyo-Kyogi Foundation (to T.I.), Japan Heart Foundation (to H.K.), IBM-Japan Heart Foundation (to H.K.), Kimura Memorial Heart Foundation (to F.K.), and Japan Research Foundation for Clinical Pharmacology (to F.K.).

Address for Reprints: Hisashi Kai, M.D., Ph.D., Third Department of Internal Medicine and Cardiovascular Research Institute, Kurume University School of Medicine, 67 Asahimachi, Kurume 830-0011, Japan. E-mail: naikai@med.kurume-u.ac.jp.

Received March 7, 2005; Accepted in revised form April 11, 2005. 

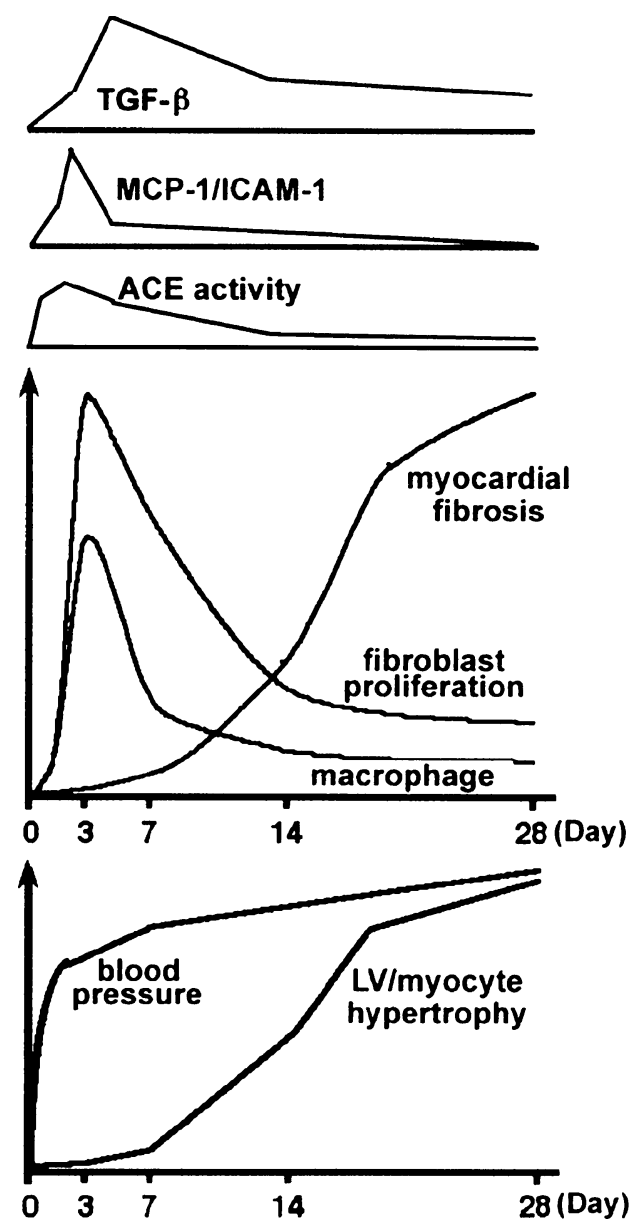

Fig. 1. Schematic diagram of the temporal changes in the early fibroinflammatory responses and the late myocardial remodeling in pressure-overloaded hearts of rats with a suprarenal aortic constriction.

with impaired systolic function (6). Therefore, appropriate therapy is desirable, but there is no available therapy specific for the prevention and improvement of diastolic dysfunction at present.

The histological features of hypertensive cardiac remodeling are myocyte hypertrophy and myocardial fibrosis. These changes are basically adaptive responses to pressure overload. However, it is considered that when hypertension persists, disproportionate LV hypertrophy and myocardial fibrosis develop and in turn result in diastolic and eventually systolic dysfunction (7). Given that recent studies have demonstrated the substantial involvement in the inflammatory process in the pathogenesis of various cardiovascular diseases, such as atherosclerosis (8), inflammatory changes may play a role in cardiac remodeling in the hypertensive heart as well. In this review, we introduce our recent studies showing that pressure overload-induced perivascular inflammation triggers myocardial fibrosis and resultant diastolic dysfunction and that the regulation of inflammation is a possible ther-

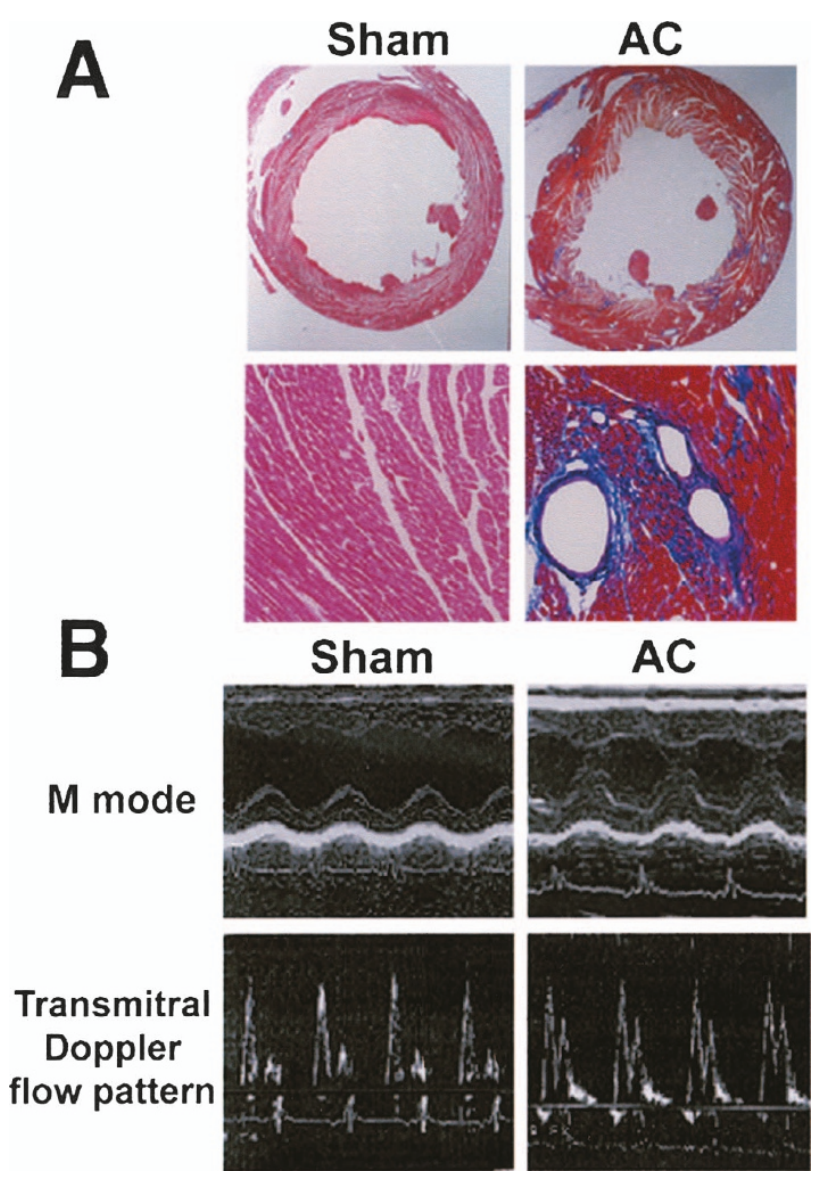

Fig. 2. A: Mallory-Azan staining at day 28. Whole sections (top) and microphotographs (bottom) of LVS obtained from rats undergoing aortic constriction (AC) or sham operation (Sham). AC induces concentric LV hypertrophy. In addition, the Azan-stained fibrotic area (stained blue) extends from the perivascular to the intermuscular interstitial spaces, which is a feature typical of reactive myocardial fibrosis. B: Echocardiographic examination at day 28. M-mode echocardiography (upper) reveals concentric hypertrophy with normal systolic function in AC rats. Decreased ratio of the early to late filling velocity (E/A) of the transmitral pulse Doppler flow pattern is observed in AC rats. Modified from Kuwahara et al. (27).

apeutic strategy for targeting diastolic dysfunction in hypertensive hearts.

\section{Myocardial Remodeling in Hypertensive Hearts}

Myocyte hypertrophy and myocardial fibrosis are important adaptive mechanisms in response to pressure overload onto the LV (9). Hypertension increases wall stress of the LV. Increased wall stress is compensated by a parallel increase in the contractile units of the cardiac myocytes, leading to myo- 
cyte hypertrophy and resultant LV wall thickening. And, an adequate degree of myocardial fibrosis prevents the ventricular deformation by increased tensile stress and transmits the force generated by hypertrophied myocytes to the entire ventricle. However, prolonged increase in cardiac work results in excessive myocyte hypertrophy and disproportionate myocardial fibrosis, which are responsible for increased myocardial stiffness and impaired pumping capacity in hypertensive patients (7).

In the early phase of hypertensive heart disease, fibrotic tissue is observed mainly in the perivascular space. Thereafter, fibrosis extends from the perivascular space to the interstitium surrounding the muscle fibers. This type of fibrosis is called "reactive fibrosis" (10). And, in patients with uncontrolled, chronic hypertension, myocyte damage and myocyte loss are induced by excessive mechanical load and sustained, inadequate activations of neurohumoral factors, including catecholamines, the renin-angiotensin-aldosterone system (RAAS), and endothelin. Consequently, patchy or massive scarring occurs in the myocardium where myocyte loss is replaced by the fibrous tissue (replacement fibrosis) (10). These changes lead to further deterioration of the LV pumping capacity.

\section{Diastolic Dysfunction in Hypertrophic Hearts}

LV diastolic dysfunction in cardiac hypertrophy results from two different mechanisms, namely, alteration in active relaxation and change in diastolic compliance $(11,12)$. Ventricular relaxation is a dynamic process that occurs during isovolumetric relaxation and early ventricular filling. The rate of ventricular relaxation is controlled primarily by the calcium uptake by the sarcoplasmic reticulum (SR) and by the sequestration of calcium from the myocytes. These processes are regulated by energy-dependent, that is, adenosine triphosphate (ATP)-dependent mechanisms, i.e., sarcoplasmic reticulum calcium-adenosine triphosphatase (SERCA) and sarcolemmal calcium pumps, respectively. In hypertrophied myocytes, the energy-dependent calcium handling is impaired by the quantitative and qualitative alterations of SERCA, phospholamban (a regulatory factor of SERCA), and ryanodine receptors (calcium-releasing channel of the $\mathrm{SR}$ ), as well as the contractile elements. And, impaired calcium handling delays active relaxation (13). Myocardial ischemia deteriorates the active relaxation as well, because the ATP depletion causes malfunction of the ATP-dependent calcium handling process.

In contrast, changes in diastolic compliance are direct consequences of exceeded chamber compliance and tissue stiffness (14). Ventricular chamber compliance depends on the ventricular diameter, and ventricular tissue stiffness is determined by the wall thickness, myocyte hypertrophy, and myocardial fibrosis. There is growing evidence that myocardial stiffening depends mainly upon myocardial fibrosis rather than LV wall thickening and myocyte hypertrophy in spontaneously hypertensive rats (SHRs) $(15,16)$, experimental renovascular hypertensive rats (17), and Dahl salt-sensitive rats (18). In these experimentally induced pressure-overload models and in patients with hypertension, there is an increase in the quantity of interstitial collagen, particularly fibrillar collagen (i.e., collagen type I and collagen type III), in the hearts (19). The accumulation of fibrillar collagen increases the inherent viscoelasticity and passive stiffness of the hypertrophied myocardium (11). Alterations of the distribution and microstructure of collagen, as well as imbalance of type I $v s$. type III collagens, affect myocardial stiffness and diastolic properties as well $(20,21)$.

\section{Models of Hypertensive Cardiac Hypertrophy with Diastolic Dysfunction}

Because of the lack of adequate animal models, it has been difficult to explore the mechanisms of hypertensive diastolic dysfunction, especially isolated diastolic dysfunction at the compensated state. Hori and his colleagues have shown that Dahl salt-sensitive rats fed a high-salt diet from 7 weeks old develop hypertension followed by LV hypertrophy with diastolic dysfunction at 13 weeks and transition to overt diastolic CHF without either LV dilatation or systolic dysfunction at 19 weeks (22). Thereafter, most rats die of CHF by 21 weeks. Accordingly, the Dahl rat model can be regarded as a model of isolated hypertensive diastolic CHF with a rapid progression and poor prognosis (22). Using the Dahl rat model, the same laboratory has been investigating the mechanisms of transition from isolated diastolic dysfunction to decompensated CHF in hypertrophic hearts $(18,23-26)$.

\section{Aortic Constriction-Induced Cardiac Hypertrophy as a New Isolated Diastolic Dysfunction Model without CHF}

Recently, we have reported that Wistar rats with a suprarenal aortic constriction (AC) are a model of cardiac hypertrophy accompanied by isolated diastolic dysfunction (27). AC induces a rapid rise in blood pressure within 1 day, and thereafter blood pressure is elevated gradually (Fig. 1). Meanwhile, concentric LV hypertrophy is established with a 2-fold increase in echocardiographic LV mass by day 28 (Fig. 2). At day 28, AC rats show normal LV fractional shortening, but decreased ratio of early to late filling velocity ( $E / A$ ratio) of the transmitral Doppler flow pattern and elevated LVEDP, indicating normal systolic but abnormal diastolic function. It is noteworthy that $\mathrm{AC}$ rats do not show typical symptoms and signs of CHF, such as orthopnea, lung or liver congestion, or excessive body weight gain, until at least 16 weeks after the induction of hypertension.

The histological features of this model are myocyte hypertrophy and reactive myocardial fibrosis (Fig. 2A). Parallel to LV hypertrophy, myocyte hypertrophy develops with a 1.6- 

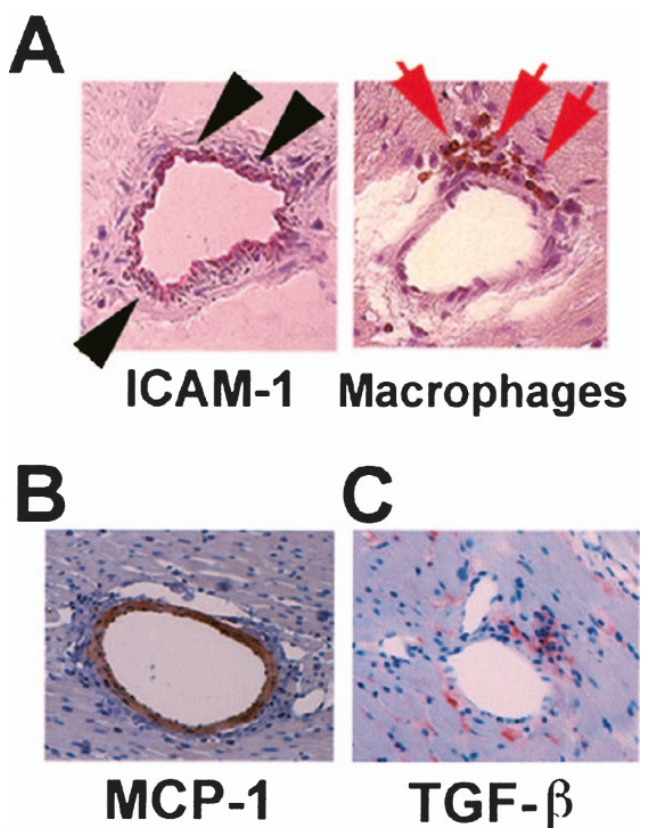

Fig. 3. Perivascular inflammatory changes in pressureoverloaded hearts. A: ICAM-1 expression (black arrowheads) is induced in the endothelium of the intramyocardial arteries with a peak at day 3 after AC (left). The serial section shows macrophage accumulation (red arrows) in the perivascular space adjacent to the arteries showing ICAM-1 expression (right). B: MCP-1 expression (brown) was induced in the entire wall of the intramyocardial arteries with a peak at day 3 after AC. C: TGF- $\beta$ expression (red) is observed in the interstitium, especially in the perivascular space, which is rich in fibroblasts with a peak at day 7. Adapted from Kuwahara et al. $(27,34,35)$.

fold increase in the myocyte diameter by day 28 (Fig. 1). Also, progressive interstitial changes are observed during LV hypertrophy: AC robustly induces fibroblast proliferation in the perivascular space at day 3 . Thereafter, fibroblast proliferation and fibrotic tissue accumulation progressively extend from the perivascular space into the adjacent intermuscular interstitium. At day 28, AC rats show perivascular fibrosis associated with interstitial fibrosis surrounding the muscle fiber bundles (Fig. 2A), which is a typical histological pattern of reactive myocardial fibrosis. And, the fibrosis area reaches to approximately $20 \%$ of the transverse area of the myocardium. There are no patchy or massive fibrotic scars replacing the areas where myocyte necrosis (replacement or reparative fibrosis) has taken place. These histological changes are similar to the myocardial remodeling seen in patients at the early stage of hypertensive heart disease (10).

Taken together with the histological features, these findings indicate that $\mathrm{AC}$ rats can be considered a model of isolated hypertensive diastolic dysfunction without overt $\mathrm{CHF}$, mim- icking the characteristics of hypertensive patients with diastolic dysfunction at the compensated state. This new model gives us the opportunity to explore the causal relation between myocardial fibrosis and diastolic dysfunction during the development of hypertensive remodeling, particularly at the early phase, because it is possible to investigate the time course of the dramatic histological changes.

\section{TGF- $\beta$-Mediated Reactive Fibrosis and Diastolic Dysfunction}

Transforming growth factor- $\beta$ (TGF- $\beta$ ) is a key molecule of tissue fibrosis in physiological and pathophysiological situations $(28,29)$. In this model, myocardial TGF- $\beta$ mRNA expression is upregulated after day 3 with a peak at day 7 (Fig. 1) (27). Thereafter, a modest elevation of TGF- $\beta$ expression remains associated with marked inductions of type I and type III collagens. Moreover, immunoreactive TGF- $\beta$ is detected in the interstitium, mainly at sites where fibroblasts are actively proliferating (Fig. 3C).

Chronic administration of anti-TGF- $\beta$-neutralizing monoclonal antibody almost abolishes fibroblast proliferation, reverses type I and type III collagen expressions to nearly normal levels, and reduces the myocardial fibrosis area by approximately $80 \%$, without affecting either blood pressure elevation or LV/myocyte hypertrophy (27). Moreover, TGF$\beta$ function blocking reverses the decreased $E / A$ ratio and elevated LVEDP to nearly normal levels, without changing systolic function. Thus, TGF- $\beta$-mediated myocardial fibrosis is suggested to play a pivotal role in the impairment of diastolic function in AC rats. The improvement of diastolic dysfunction induced by blocking TGF- $\beta$ may be attributable mainly to a reduction in the collagen contents responsible for myocardial stiffening. Because intense perivascular fibrosis impairs oxygen diffusion to hypertrophied myocardium, it is also possible that the attenuation of perivascular fibrosis would improve relative myocardial ischemia and subsequently attenuate diastolic dysfunction.

\section{Perivascular Inflammation and Hypertensive Remodeling}

Atherosclerosis is now widely accepted as a chronic vascular inflammation disorder (30). A pivotal role in this process is represented by circulating inflammatory cells that attach to the endothelium and migrate into the vascular lesions by interacting with adhesion molecules, such as intercellular adhesion molecule-1 (ICAM-1) and vascular adhesion molecule-1, and chemokines secreted by the activated vessel wall, such as monocyte chemoattractant protein-1 (MCP-1). Transmigration of macrophages is the earliest and most significant inflammatory event in vascular lesion formation because it induces production of inflammatory cytokines and growth factors, which in turn activate further vascular inflammation and lesion formation. 


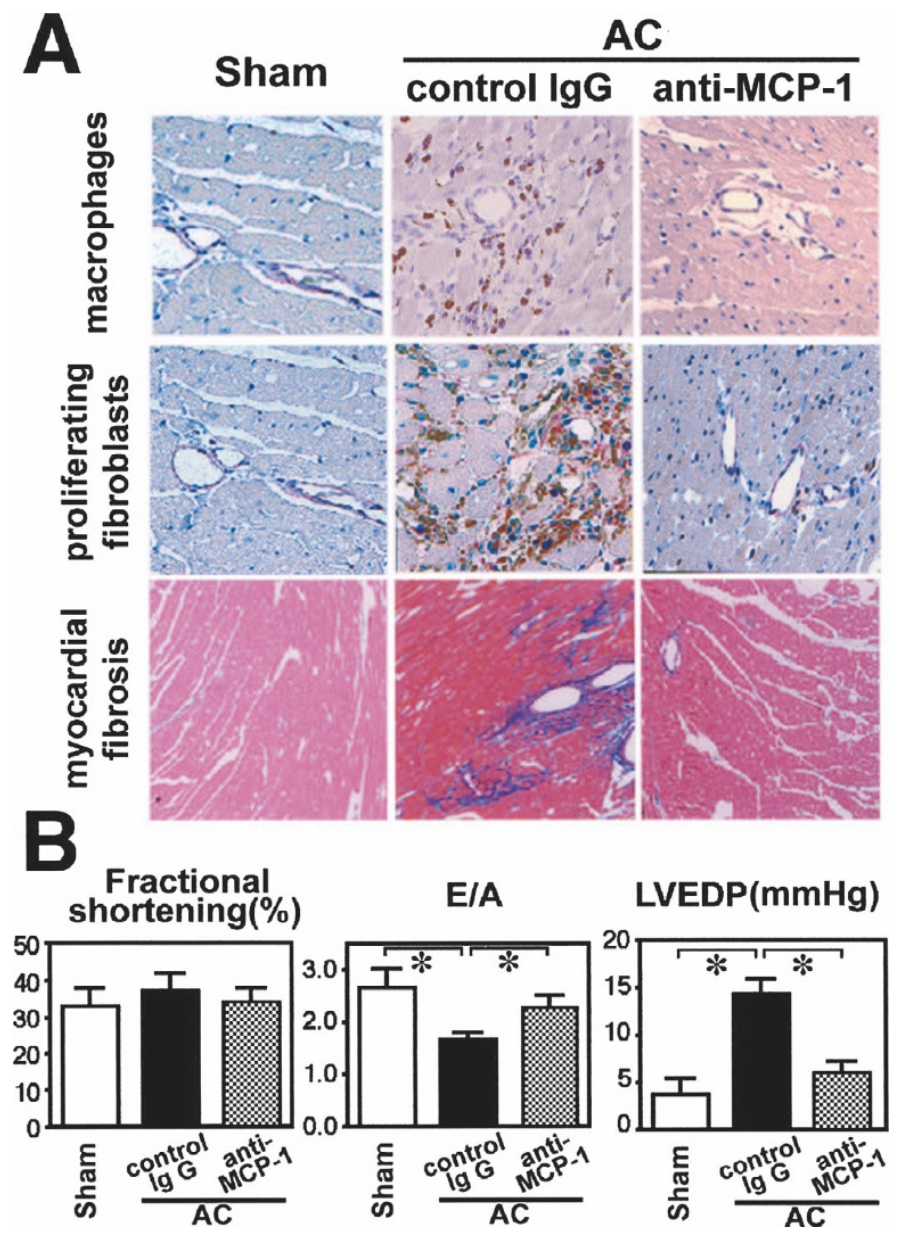

Fig. 4. Effects of MCP-1 function blocking. A: Perivascular accumulations of macrophages (brown, top) and BrdU ${ }^{+}$(brown)/ vimentin ${ }^{+}$(red) proliferating fibroblasts (middle) at day 3 are almost abolished in rats with an aortic constriction (AC) by antiMCP-1 neutralizing monoclonal antibody (anti-MCP-1). At day 28, AC-induced myocardial fibrosis (blue) is remarkably prevented by blocking the MCP-1 function (bottom). B: Left ventricular fractional shortening (left) and the ratio of the early to late filling velocity (E/A) of transmitral flow velocity (middle) are assessed on the basis of M-mode and pulse Doppler echocardiography, respectively, at day 28. Left ventricular end-diastolic pressure (LVEDP) is measured using a micromanometer-tipped catheter at day 28 (right). Sham, sham-operated rats; control IgG, non-immunized IgG. Modified from Kuwahara et al. (34).

\section{Perivascular Inflammation in Hypertensive Hearts}

Earlier studies reported that inflammatory cells, particularly macrophages, and fibroblasts showing proliferation and collagen production are found in the perivascular space of hypertrophied hearts of SHRs and experimental renovascular hypertensive rats $(31,32)$. Also, ICAM-1 expression was observed in endothelial cells of the intramyocardial arteries in these models (33). These observations raise an attractive hypothesis that the inflammatory process plays a role in hypertensive myocardial remodeling. However, it has not been determined whether the inflammatory changes are causally linked to the fibrotic process or whether they are the result of hypertensive end-organ damage.

In AC rats, an initial, rapid pressure rise triggers a series of inflammatory changes in the intramyocardial arterial wall (Fig. 1). At days 1-7, ICAM-1 and MCP-1 are transiently induced in the endothelium and the media of the intramyocardial arteries, respectively (Fig. 3). Moreover, macrophages accumulate predominantly in the perivascular space adjacent to the arteries expressing ICAM-1 and MCP-1 (Fig. 3A and $\mathrm{B})$. It is noteworthy that the ICAM-1 and MCP-1 inductions and macrophage infiltration are transient events, despite the fact that the blood pressure remains elevated (Fig. 1). Also, it is of interest that macrophages and proliferating fibroblasts show similar spatial and temporal distributions.

MCP-1 function blocking using a neutralizing monoclonal antibody almost abolishes macrophage infiltration and remarkably reduces TGF- $\beta$ induction and fibroblast proliferation, without changing blood pressure, in AC rats (Fig. 4A) (34). Moreover, AC-induced perivascular and interstitial 


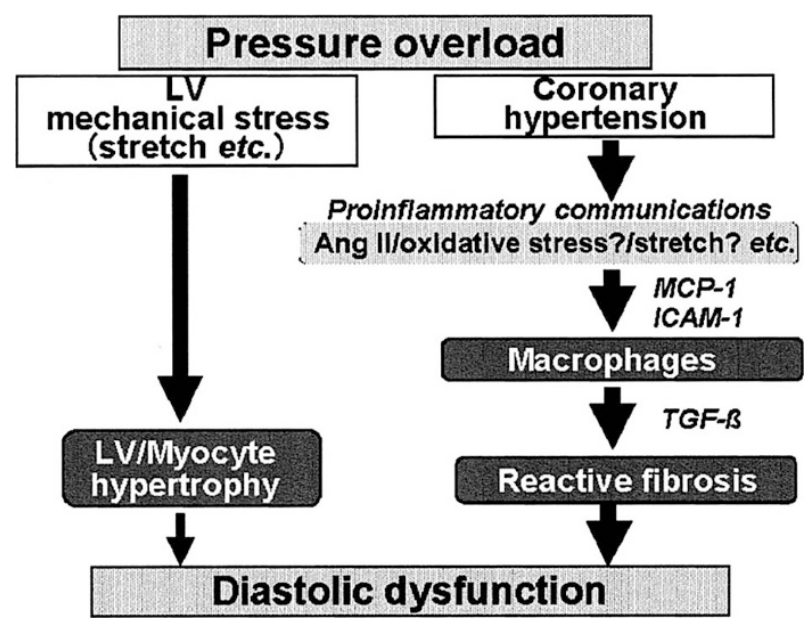

Fig. 5. Schematic showing the role of the perivascular inflammatory process in myocardial remodeling and diastolic dysfunction in pressure-overloaded hearts.

fibrosis, but not myocyte/LV hypertrophy, are prevented by blocking MCP-1 (Fig. 4A). Similar results are obtained when macrophage infiltration is prevented by ICAM-1 function blocking (35). Thus, it is suggested that macrophages are recruited by the coordinated effect of MCP-1 and ICAM-1, then transmigrate to the perivascular space, and in turn activate the cascade of myocardial fibrosis. Macrophages produce not only TGF- $\beta$ (36) but also profibrotic cytokines (37, 38 ). TGF- $\beta$ induces a phenotypic change from fibroblasts to myofibroblasts. Activated fibroblasts and myofibroblasts then express TGF- $\beta$ and other profibrotic substances, which in turn self-amplify ongoing fibrotic tissue formation (39). Moreover, MCP-1 function blocking not only prevents myocardial fibrosis but also ameliorates diastolic dysfunction without affecting systolic function in AC rats (Fig. 4B) (34). Accordingly, it is suggested that macrophage-mediated perivascular inflammation is a key event for triggering both the early fibrotic changes (fibroblast proliferation and TGF- $\beta$ induction) and the late reactive fibrosis and diastolic dysfunction in pressure-overloaded hearts.

The AC rat model is characterized by acute hypertension. Thus, the observed phenomena may reflect an acute reaction of the hearts in response to sudden increase in blood pressure, but not a reaction to chronic hypertension. Very recently, it has been shown that increased blood pressure variability is an independent risk factor for cardiovascular events in elderly hypertensive patients (40). Accordingly, it is possible that in hypertensive patients with exaggerated blood pressure variability, repeated, abrupt pressure elevations induce repetitive and chronic perivascular inflammation and in turn promote cardiovascular damages.

\section{Mechanisms of Perivascular Inflammation}

\section{Proinflammatory Communications around the Coronary Arterial Wall}

The trigger element of ICAM-1 and MCP-1 inductions remains undetermined. AC rats did not show significant changes in plasma rennin activity (27), serum angiotensinconverting enzyme (ACE) activity (41), and the circulating levels of angiotensin II and aldosterone (unpublished data), suggesting that the systemic RAAS is not likely to play a role in cardiac remodeling. In addition, AC induces perivascular fibrosis, but not myocyte hypertrophy, in the right ventricular (RV) free-wall (34). The increase in vascular wall tension induced by hypertension is distributed throughout the whole coronary artery trees of both LV and RV, although RV is not exposed to high pressure load. Thus, it is suggested that coronary arterial hypertension contributes to reactive myocardial fibrosis, whereas myocyte hypertrophy is attributable to LV systolic mechanical overload.

There is emerging evidence that arterial hemodynamic stress could activate the proinflammatory communications around the arterial wall (38), which in turn could induce inflammatory mediators, including MCP-1 (Fig. 5). First, an increase in arterial pressure is itself implicated as a strong signal for activation of MCP-1 induction in the hypertensive aortic wall (42), suggesting that mechanical strain of the arterial wall directly upregulates MCP-1 (43). Second, reactive oxygen species and inflammatory cytokines and growth factors, such as TGF- $\beta$, platelet-derived growth factor, and angiotensin II, are produced in the hypertensive arterial wall (44). These factors can upregulate MCP-1 expression (37). Taken together, these findings suggest that MCP-1 induction in pressure-overloaded hearts may occur as the result of an intricate interplay between the mechanical stimulus and the changes in proinflammatory factors derived from the neuroendocrine system, infiltrating inflammatory cells or vessel wall cells.

\section{The Role of the Tissue Angiotensin System}

$\mathrm{AC}$-induced abrupt pressure rise triggers a rapid and transient activation of tissue ACE, but not serum ACE, within day 1 with a peak at day 3 (Fig. 1) (41). And, the time course of the ACE activation is consistent with those of ICAM-1 and MCP1 inductions as well as macrophage infiltration. Moreover, a subdepressor dose of candesartan, an angiotensin II type I receptor (AT1) antagonist, not only suppresses the early inflammatory (MCP-1 induction and macrophage infiltration) and fibrotic (TGF- $\beta$ induction and fibroblast proliferation) changes but also reduces the late reactive myocardial fibrosis, without effects on myocyte/LV hypertrophy, in AC rats (41, 45). Angiotensin II has been shown to support inflammatory cell transmigration in an AT1 receptor-dependent manner, but not in an arterial pressure-independent manner $(46,47)$. Also, 
angiotensin II induces MCP-1 expression in vascular smooth muscle cells (48) and macrophages (49) and upregulates TGF- $\beta$ and the extracellular matrices in cardiac myocytes and fibroblasts (50). In this model, AC-induced cardiac ACE activation is not affected by AT1 receptor blockade (41). These results suggest that activation of the tissue angiotensin system is an event occurring upstream of the early fibroinflammatory changes and angiotensin II is one of the major paracrine/ endocrine proinflammatory factors responsible for reactive fibrosis in pressure-overloaded hearts.

There is increasing evidence suggesting that AT1 receptor and angiotensin II type 2 (AT2) receptor play reciprocal roles in the development of pressure-overload-induced cardiac remodeling $(51,52)$. Thus, the roles of AT2 receptors in perivascular inflammation and reactive fibrosis should be elucidated in a future study. Also, recent studies have shown that aldosterone is a strong inducer of vascular inflammation and myocardial fibrosis (53). It has been shown that aldosteroneinduced vascular inflammation and myocardial fibrosis are associated with high salt intake. However, we cannot deny the possibility that aldosterone, especially locally generated aldosterone, is involved in the inflammatory process in this model. Further investigations will be needed to address this issue.

\section{Conclusions}

Based on our recent studies, we suggest the novel concept that hypertensive myocardial fibrosis and diastolic dysfunction are another model of vascular inflammation. Targeting inflammatory processes, including angiotensin II, ICAM-1, and MCP-1, may be a new strategy for prevention and treatment of diastolic dysfunction in hypertensive hearts.

\section{Acknowledgements}

We thank Mss. K. Kimura, K. Moriyama, Y. Yoshida, K. Yoshida, and R. Fujiyoshi for their excellent technical assistance.

\section{References}

1. Vasan RS, Benjamin EJ, Levy D: Prevalence, clinical features and prognosis of diastolic heart failure: an epidemiologic perspective. J Am Coll Cardiol 1995; 26: 1565-1574.

2. Vasan RS, Larson MG, Benjamin EJ, Evans JC, Reiss CK, Levy D: Congestive heart failure in subjects with normal versus reduced left ventricular ejection fraction: prevalence and mortality in a population-based cohort. $\mathrm{J} \mathrm{Am} \mathrm{Coll} \mathrm{Car-}$ diol 1999; 33: 1948-1955.

3. Tsuchihashi M, Tsutsui H, Kodama K, Kasagi F, Takeshita A: Clinical characteristics and prognosis of hospitalized patients with congestive heart failure - a study in Fukuoka, Japan. Jpn Circ J 2000; 64: 953-995.

4. Tsutsui H, Tsuchihashi M, Takeshita A: Mortality and readmission of hospitalized patients with congestive heart failure and preserved versus depressed systolic function. Am J Cardiol 2001; 88: 530-533.
5. Mann DL: Mechanisms and models in heart failure. Circulation 1999; 100: 999-1008.

6. Senni M, Tribouilloy CM, Rodeheffer RJ, et al: Congestive heart failure in the community: a study of all incident cases in Olmsted County, Minnesota, in 1991. Circulation 1998; 98: 2282-2289.

7. Weber KT, Brilla CG: Pathological hypertrophy and cardiac interstitium. Fibrosis and renin-angiotensin-aldosterone system. Circulation 1991; 83: 1849-1865.

8. Brasier AR, Recinos A III, Eledrisi MS: Vascular inflammation and the renin-angiotensin system. Arterioscler Thromb Vasc Biol 2002; 22: 1257-1266.

9. Brilla CG, Weber KT: Reactive and reparative myocardial fibrosis in arterial hypertension in the rats. Cardiovasc Res 1992; 26: 671-677.

10. Weber KT, Brilla CG, Janicki JS: Myocardial fibrosis: functional significance and regulatory factors. Cardiovasc Res 1993; 27: 341-348.

11. Jalil JE, Doering CW, Janicki JS, PicK R, Shroff SG, Weber KT: Fibrillar collagen and myocardial stiffness in intact hypertrophied rat left ventricle. Circ Res 1989; 64: 1041-1050.

12. Swynghedauw B: Molecular mechanisms of myocardial remodeling. Physiol Rev 1999; 79: 215-262.

13. Zile RM, Brutsart DL: New concepts in diastolic dysfunction and diasolic heart failure: part II. Causal mechanisms and treatment. Circulation 2002; 105: 1503-1508.

14. Mandinov L, Eberli FR, Seiler C, Hess OM: Diastolic heart failure. Cardiovasc Res 2000; 45: 813-825.

15. Narayan S, Janicki JS, Shroff SG, Pick R, Weber KT: Myocardial collagen and mechanics after preventing hypertrophy in hypertensive rats. Am J Hypertens 1989; 2: 675-682.

16. Conrad CH, Brooks WW, Hayes JA, Sen S, Robinson KG, Bing OL: Myocardial fibrosis and stiffness with hypertrophy and heart failure in spontaneously hypertensive rats. Circulation 1995; 91: 161-170.

17. Matsubara LS, Matsubara BB, Okoshi MP, Cicogna AC, Janicki JS: Alterations in myocardial collagen content affect rat papillary muscle function. Am J Physiol 2000; 279: H1534-H1539.

18. Yamamoto K, Masuyama T, Sakata Y, et al: Myocardial stiffness is determined by ventricular fibrosis, but not by compensatory or excessive hypertrophy in hypertensive heart. Cardiovasc Res 2002; 55: 76-82.

19. Burlew BS, Weber KT: Cardiac fibrosis as a cause of diastolic dysfunction. Herz 2002; 27: 92-98.

20. Villarreal FJ, Dillmann WH: Cardiac hypertrophy-induced changes in mRNA levels for TGF- $\beta 1$, fibronectin and collagen. Am J Physiol 1992; 262: H1861-H1866.

21. Kato S, Spinale FG, Tanaka R, Johnson W, Cooper G IV, Zile MR: Inhibition of collagen cross-linking: effects on fibrillar collagen and ventricular diastolic function. $\mathrm{Am} \mathrm{J}$ Physiol 1995; 269: H863-H868.

22. Doi R, Masuyama T, Yamamoto K, et al: Development of different phenotypes of hypertensive heart failure: systolic versus diastolic failure in Dahl salt-sensitive rats. J Hypertens 2000; 18: 111-120.

23. Yamamoto K, Masuyama T, Sakata Y, et al: Local neurohumoral regulation in the transition to isolated diastolic heart failure in hypertensive heart disease: absence of AT1 
receptor downregulation and 'overdrive' of endothelin system. Cardiovasc Res 2000; 46: 421-432.

24. Yamamoto K, Masuyama T, Sakata Y, Nishikawa N, Mano T, Hori M: Prevention of diastolic heart failure by endothelin type A receptor antagonist through inhibition of ventricular structural remodeling in hypertensive heart. $J$ Hypertens 2002; 20: 753-761.

25. Nishikawa N, Yamamoto K, Sakata Y, et al: Differential activation of matrix metalloproteinases in heart failure with and without ventricular dilatation. Cardiovasc Res 2003; 57: 766-774.

26. Sakata Y, Yamamoto K, Mano T, et al: Activation of matrix metalloproteinases precedes left ventricular remodeling in hypertensive heart failure rats: its inhibition as a primary effect of angiotensin-converting enzyme inhibitor. Circulation 2004; 109: 2143-2149.

27. Kuwahara F, Kai H, Tokuda K, et al: Transforming growth factor- $\beta$ function blocking prevents myocardial fibrosis and diastolic dysfunction in pressure-overloaded rats. Circulation 2002; 106: 130-135.

28. Tahira Y, Fukuda N, Endo M, et al: Transforming growth factor- $\beta$ expression in cardiovascular organs in stroke-prone spontaneously hypertensive rats with the development of hypertension. Hypertens Res 2002; 25: 911-918.

29. Saito K, Ishizaka N, Aizawa T, et al: Role of aberrant iron homeostasis in the upregulation of transforming growth factor- $\beta 1$ in the kidney of angiotensin II-induced hypertensive rats. Hypertens Res 2004; 27: 599-607.

30. Ross R: Atherosclerosis: an inflammatory disease. $N$ Engl J Med 1999; 340: 115-126.

31. Hinglais N, Heudes D, Nicoletti A: Colocalization of myocardial fibrosis and inflammatory cells in rats. Lab Invest 1994; 70: 286-294.

32. Nicoletti A, Heudes D, Mandet C, Hinglais N, Bariety J, Michel JB: Inflammatory cells and myocardial fibrosis: spatial and temporal distribution in renovascular hypertensive rats. Cardiovasc Res 1996; 32: 1096-1107.

33. Komatsu S, Panes J, Russel JM, Anderson DC, Miyasaka M, Granger DN: Effects of chronic arterial hypertension on constitutive and induced intracellular adhesion molecule-1 expression in vivo. Hypertension 1997; 29: 683-689.

34. Kuwahara F, Kai H, Tokuda K, et al: Hypertensive myocardial fibrosis and diastolic dysfunction-another model of inflammation-. Hypertension 2004; 43: 739-745.

35. Kuwahara F, Kai H, Tokuda K, et al: Roles of intercellular adhesion molecule-1 in hypertensive cardiac remodeling. Hypertension 2003; 41: 819-823.

36. Border WA, Nobel NA: Transforming growth factor- $\beta$ in tissue fibrosis. N Engl J Med 1994; 331: 1286-1292.

37. Reap TJ, Groot PHE: Chemokines and atherosclerosis. Atherosclerosis 1999; 147: 213-225.

38. Nicoletti A, Michel JB: Cardiac fibrosis and inflammation: interaction with hemodynamic and hormonal factors. Cardiovasc Res 1999; 41: 532-543.

39. Desmoulière A, Geinoz A, Gabbiani F, Gabbiani G: Transforming growth factor- $\beta 1$ induces $\alpha$-smooth muscle actin expression in granulation tissue myofibroblasts and in qui- escent and growing cultured fibroblasts. J Biol Chem 1993; 122: 103-111.

40. Eto M, Toba K, Akishita M, et al: Impact of blood pressure variability on cardiovascular events in elderly patients with hypertension. Hypertens Res 2005; 28: 1-7.

41. Tokuda K, Kai H, Kuwahara F, et al: Pressure-independent effects of angiotensin II on hypertensive myocardial fibrosis. Hypertension 2004; 43: 499-503.

42. Capers Q IV, Alexander RW, Lou P, et al: Monocyte chemoattractant protein-1 expression in aortic tissues of hypertensive rats. Hypertension 1997; 30: 1397-1402.

43. Jiang MJ, Yu YJ, Chen YL, Lee YM, Hung LS: Cyclic strain stimulates monocyte chemotactic protein-1 mRNA expression in smooth muscle cells. J Cell Biochem 1999; 76: 303-310.

44. Alexander RW: Hypertension and the pathogenesis of atherosclerosis. Oxidative stress and the mediation of arterial inflammatory response: a new perspectives. Hypertension 1995; 25: 155-161.

45. Tokuda K, Kai H, Kuwahara F, Imaizumi T: Sub-depressor dose of angiotensin type-1 receptor blocker inhibits TGF- $\beta$ mediated perivascular fibrosis in hypertensive rat hearts. $J$ Cardiovasc Pharmacol 2003; 42 (Suppl): S61-S65.

46. Mervaala EMA, Müller DN, Park J-K, et al: Monocyte infiltration and adhesion molecules in a rat model of high human renin hypertension. Hypertension 1999; 33: 389395.

47. Pastore L, Tessitore A, Martinotti S, et al: Angiotensin II stimulates intracellular adhesion molecule-1 (ICAM-1) expression by human vascular endothelial cells and increases soluble ICAM-1 release in vivo. Circulation 1999; 100: 1646-1652.

48. Chen XL, Tummala PE, Olbrych MT, Alexander RW, Medford RM: Angiotensin II induces monocyte chemoattractant protein-1 gene expression in rat vascular smooth muscle cells. Circ Res 1998; 83: 952-959.

49. Hernandez-Presa M, Bustos C, Ortego M, et al: Angiotensin-converting enzyme inhibition prevents arterial nuclear factor-kappa B activation, monocyte chemoattractant protein-1 expression, and macrophage infiltration in a rabbit model of early accelerated atherosclerosis. Circulation 1997; 95: 1532-1541.

50. Border WA, Ruoslahti E: Transforming growth factor- $\beta$ in disease: the dark side of tissue repair. J Clin Invest 1992; 90: 1-7.

51. Kurisu S, Ozono R, Oshima T, et al: Cardiac angiotensin II type 2 receptor activates the kinin/NO system and inhibits fibrosis. Hypertension 2003; 41: 99-107.

52. Mukawa H, Toki Y, Miyazaki Y, Matsui H, Okumura K, Ito T: Angiotensin II type 2 receptor blockade partially negates antihypertrophic effects of type 1 receptor blockade on pressure-overload rat cardiac hypertrophy. Hypertens Res 2003; 26: 89-95.

53. Sato A, Saruta T: Aldosterone-induced organ damage: plasma aldosterone level and inappropriate salt status. Hypertens Res 2004; 27: 303-310. 